Oikos 117: 1840-1846, 2008

doi: $10.1111 / \mathrm{j} .1600-0706.2008 .17028 . x$,

(C) 2008 The Authors. Journal compilation (C) 2008 Oikos

Subject Editor: Matt Ayres. Accepted 30 June 2008

\title{
Pesticide exposure strongly enhances parasite virulence in an invertebrate host model
}

\author{
Anja Coors, Ellen Decaestecker, Mieke Jansen and Luc De Meester
}

A. Coors (a.coors@ect.de), E. Decaestecker, M. Jansen and L. De Meester, Lab. of Aquatic Ecology and Evolutionary Biology, Katholieke Univ. Leuven, Ch. De Bériotstraat 32, BE-3000 Leuven, Belgium. Present address for AC: ECT Oekotoxikologie GmbH, Boettgerstr. 2-14, DE65439 Flörsheim a. M., Germany.

\begin{abstract}
Parasites are a common and constant threat to organisms at all levels of phyla. The virulence of a parasite, defined as the impact on survival and reproduction of its host, depends on the specific host-parasite combination and can also be influenced by environmental conditions. Environmental pollution might be an additional factor influencing hostparasite interactions. We here aimed to test whether the combined stress of pollutant exposure and parasite challenge results in stronger impacts on host organisms than expected from the single stressors applied alone. We used the water flea Daphnia magna and two of its endoparasites, the bacterium Pasteuria ramosa and the microsporidium Flabelliforma magnivora, as invertebrate host-parasite models. For each parasite, we tested in a full-factorial design for interactions between parasitism and pollution using the neurotoxic pesticide carbaryl as a model substance. Sublethal concentrations of the pesticide synergistically enhanced the virulence of both parasites by increasing host mortality. Furthermore, host castration induced by $P$. ramosa was accelerated by carbaryl exposure. These effects likely reflect decreased host resistance due to direct or indirect immunosuppressive activity of carbaryl. The present study provides experimental evidence that the in vivo development of infectious diseases can be influenced by a pesticide at environmentally realistic concentrations. This implies that host-parasite interactions and subsequently co-evolution might be influenced by environmental pollution at toxicant concentrations being sublethal to parasite-free hosts. Standard toxicity testing as employed in the current way of conducting ecological risk assessments for anthropogenic substances does not consider natural antagonists such as infectious diseases, and thereby likely underestimates the impact these substances may pose to natural populations in the environment.
\end{abstract}

In all natural populations, individuals face multiple antagonists such as predators, competitors and parasites (Thompson 2005). In addition to the pressure posed by natural antagonists, environmental pollution can be considered as an abiotic stressor of anthropogenic origin, frequently occurring in many types of habitats and having strong impacts on natural communities (Rohr et al. 2006). Environmental pollutants may interact with natural stressors leading to effects not predictable from the ecology of the involved organisms nor from the inherent toxicity of the pollutant. Relyea and Mills (2001) substantiated this concern by reporting increased lethality of the pesticide carbaryl when gray treefrog tadpoles exposed to carbaryl were additionally stressed by the presence of a caged predator.

Next to predators, parasites are another common biotic antagonist posing a constant threat to individuals at all levels of phyla (Minchella and Scott 1991). Parasite virulence, defined as impact of the parasite on host survival and reproduction (Bull 1994, Ebert 1994), is an important factor driving host-parasite dynamics (Anderson and May 1979). Hence, small changes in parasite virulence due to synergistic interaction with environmental stress may distinctly alter the ecology and evolution of host-parasite interactions. Virulence depends on host and parasite genotype (Carius et al. 2001), transmission routes (Restif and Kaltz 2006), and environmental conditions. Abiotic stress factors such as anoxia and food depletion have been shown to enhance the virulence of a trematode parasite in a molluscan host (Jokela et al. 2005). Likewise, interactions between parasitic diseases and toxic pollutants may result in stronger impacts of the parasite, the toxicant or both. Thus, effects of the toxicant on the host might be stronger or occur at lower concentrations than effects of the toxicant observed in the absence of the parasite. Furthermore, enhanced parasite impact due to toxicant exposure might involve increased parasite virulence and/or decreased disease resistance of the host as reflected in a higher infection rate or a faster disease development. The underlying processes of such effects might either be a general stress-induced decrease in host condition or a direct immunotoxic activity of the pollutant. Such impairments of immunocompetence and associated decreased resistance to parasites caused by environmental pollution are an issue of enduring and still 
unresolved concern for the health of wildlife and human populations (Street and Sharma 1975, Galloway and Depledge 2001, Koller 2001, Descotes 2006).

The present study examines the interaction between a model pollutant and parasitic diseases in an invertebrate host model. Parasitic disease is represented by two parasites from two different taxonomic classes and with different modes of transmission. We used the methyl carbamate carbaryl as model substance, representative for a class of pesticides (methyl carbamates and organophosphorus insecticides) with the same mode of action and widely applied in agriculture. The neurotoxic activity of these pesticides is due to the inhibition of acetylcholinesterase, an essential enzyme that deactivates the ubiquitous neurotransmitter acetylcholine. Our host model Daphnia magna is well characterized and has often been used to study host-parasite interactions such as co-evolution and genotype-related specificity of virulence and resistance (Ebert et al. 2000, 2004, Carius et al. 2001, Mitchell et al. 2004, Decaestecker et al. 2007, Duncan and Little 2007). The crustacean D. magna is a keystone species in freshwater ecosystems and known to host a broad range of parasites, among them the two parasites used in the present study, the bacterium Pasteuria ramosa and the microsporidium Flabelliforma magnivora (Green 1974, Mangin et al. 1995, Stirnadel and Ebert 1997, Ebert et al. 2001). Here, we performed two full-factorial experiments with parasite and pesticide exposure as factors and evaluated the effects on both host survival and parameters related to host fecundity.

\section{Material and methods}

The potential interaction of carbaryl exposure and parasites was investigated in two independent full-factorial experiments using two different clones of $D$. magna and two different parasites. The experiments were similar with regard to pesticide exposure. Experiments and stock culturing of the D. magna clonal lineages were conducted in environmental chambers at $19^{\circ} \mathrm{C} \pm 1$ and diffuse light (16:8 h light-dark cycle) using artificial medium (ADaM, Klüttgen et al. 1994). Clonal lineages were cultured in the laboratory for several generations under standardized conditions before experiments were started with neonates ( $<24 \mathrm{~h}$ old) from 3 rd brood (experiment 1$)$ or 2 nd brood (experiment 2).

Carbaryl (1-naphthyl methylcarbamate, CAS 63-25-2, purity $99.8 \%$, Sigma-Aldrich, Germany) stock solutions were prepared in ethanol (p.a.) and the final solvent concentration $\left(0.05 \mathrm{ml} \mathrm{l}^{-1}\right)$ was the same in all treatments. This ethanol concentration was in many preliminary experiments shown to have no effect on D. magna (data not shown). The acute toxicity of carbaryl towards $D$. magna is characterized by a very steep dose response curve $\left(100 \%\right.$ mortality at $11.3 \mu \mathrm{g} \mathrm{l}^{-1}$ and no significant effects at $7.0 \mathrm{~g} \mathrm{l}^{-1}$; Coors unpubl.). Based on this information, two carbaryl concentrations $\left(5.6\right.$ and $8.0 \mu \mathrm{g} \mathrm{l}^{-1}$ ) and a solvent control (ethanol-only) were tested in both experiments, always in the absence or in the presence of the respective parasite. In both experiments, the medium (and thus the carbaryl concentration) was renewed every second day, and carbaryl exposure was maintained through the entire 18-day experiments. All D. magna were fed daily with Scenedesmus obliquus. Food level in experiment 1 increased from $1.0 \times$ $10^{5}$ cells $\mathrm{ml}^{-1}$ (equal to $0.75 \mathrm{mg} \mathrm{C}^{-1}$ ) during day $0-3$ to finally $1.2 \times 10^{5}$ cells $\mathrm{ml}^{-1}$ from day 10 onwards. In experiment 2 , the food level increased from $2.0 \times 10^{5}$ cells $\mathrm{ml}^{-1}$ during day $0-10$ to $4.0 \times 10^{5}$ cells $\mathrm{ml}^{-1}$ thereafter, which was identical to the food regime of the stock cultures.

\section{Experiment 1}

We used the bacterial endoparasite Pasteuria ramosa in experiment 1 . This experiment was conducted with about 450 experimental animals in total, split into five replicates for each of the six treatments and each replicate consisting out of 13-16 (mostly 15) individual D. magna kept together as an experimental group during the whole experiment.

Pasteuria ramosa irreversibly castrates the usually parthenogenetically reproducing host within 5 to 15 days after infection, thereby channelling energy resources to the development of new parasite transmission stages (Ebert et al. 2004). Transmission occurs horizontally by endospores released only from decaying hosts (Ebert et al. 2004), which enables controlled laboratory experiments with defined endospore doses. To obtain $P$. ramosa endospores, $D$. magna neonates were exposed in the laboratory to the sediment from a pond in Heverlee, Belgium (OM2, Abdij van 't Park, 50 $\left.51^{\prime} 48^{\prime \prime} \mathrm{N}, 4^{\circ} 43^{\prime} 17^{\prime \prime} \mathrm{E}\right)$, which is characterized by Pasteuria epidemics (Decaestecker et al. 2004, 2007). The same D. magna clone, which was isolated from a different pond than the sediment, was used to pick up $P$. ramosa and in the experiment. After about 28 days, Pasteuria-infected hosts were ground up and the resulting solutions were filtered ( $60 \mu \mathrm{m}$ nylon filters, Millipore) and diluted with ADaM. Solutions for non-parasite challenged so-called placebo treatments were prepared in the same way with healthy stock culture daphnids and contained an equal amount of ground daphnid tissue.

In treatments with $P$. ramosa challenge, $1 \mathrm{ml}$ of a solution containing $10^{6}$ mature $P$. ramosa endospores was added to $19 \mathrm{ml}$ fresh medium at day 0 and at day 2, whereas all other treatments received instead $1 \mathrm{ml}$ of the placebo solution. Challenge by $P$. ramosa endospores stopped at day 4, when daphnids were transferred from the vessels with $20 \mathrm{ml}$ medium to larger vessels containing $500 \mathrm{ml}$ medium. Survival was checked daily and occurrence and time to castration were recorded for each surviving individual. Castration (characterized by the absence of eggs in the brood chamber and of egg development in the ovaries) was later confirmed to be a reliable sign of $P$. ramosa infection by microscopic inspection. Therefore, castration rates at the end of the experiment correspond to infection rates. A smaller-scaled pre-test had previously indicated synergistic effects between carbaryl exposure and $P$. ramosa challenge on mortality and infection rate, but not on number of offspring in non-castrated hosts (data not shown). Therefore, the present main experiment was designed to obtain data appropriate for ANOVA for the binary endpoints castration and mortality by replicated grouped exposure of many animals. As the Pasteuria effect on host fecundity is realized by full castration of the Daphnia host, and thus 
intrinsically shows a strong parasite effect on host fecundity, we did not additionally count the total number of offspring produced by the host.

\section{Experiment 2}

The microsporidian endoparasite Flabelliforma magnivora was used in experiment 2. F. magnivora (formerly named Tuzetia sp., Ebert 2005) is a vertically transmitted parasite, thus the infection is directly transferred from the mother to the offspring (Mangin et al. 1995, Ebert et al. 2000). The efficiency of the vertical transmission of this parasite is close to $100 \%$ (Mangin et al. 1995). By mass-culturing of an infected clone (hatched in the laboratory from a dormant egg) and visually scanning hundreds of offspring, we were able to obtain an individual not carrying the parasite, which was further cultured as a separate clonal lineage. In this way, we obtained cultures of infected and uninfected hosts of the same genotype. Genetic identity of the clonal lineages was confirmed by repeated genotyping at four polymorphic allozyme loci. Thus, these two clonal lineages represent presence or absence of the factor 'parasite' in an isogenic D. magna population.

The experiment was conducted with ten replicates for each of the six treatments (presence or absence of parasite crossed with two carbaryl levels and a solvent control), each replicate consisting of a $D$. magna female kept singly in $50 \mathrm{ml}$ medium. During daily routines, survival was checked and offspring removed and counted. Microscopic inspection at the end of the experiment confirmed that all experimental animals from the parasite treatments were infected by $F$. magnivora, whereas those from the nonparasite treatments were not infected.

\section{Statistical analysis}

Two-way ANOVAs were performed with mortality rates per vessel for experiment 1 and number of living offspring per surviving female for experiment 2. Data on castration from experiment 1 were analysed by one-way ANOVAs within parasite treatments. This was due to the fact that castration is exclusively caused by the parasite and the resulting lack of variation in the placebo treatments does not allow a two-way ANOVA. All data analysed by ANOVA (Statistica, ver. 7.1, 2006) complied with assumptions of normality and homoscedasticity without transformation. Binary mortality data from experiment 2 were analysed by pairwise comparisons within each treatment using Fisher exact tests with Bonferroni correction (in total nine tests).

\section{Results}

\section{Experiment 1}

Mortality occurred mainly until day 10 (end of juvenile phase) and was the greatest $(25 \%)$ in the $8.0 \mu \mathrm{g} \mathrm{l}^{-1}$ carbaryl/parasite treatment (Fig. 1A). Carbaryl exposure and parasite challenge acted synergistically on mortality, as reflected by the statistically significant interaction term
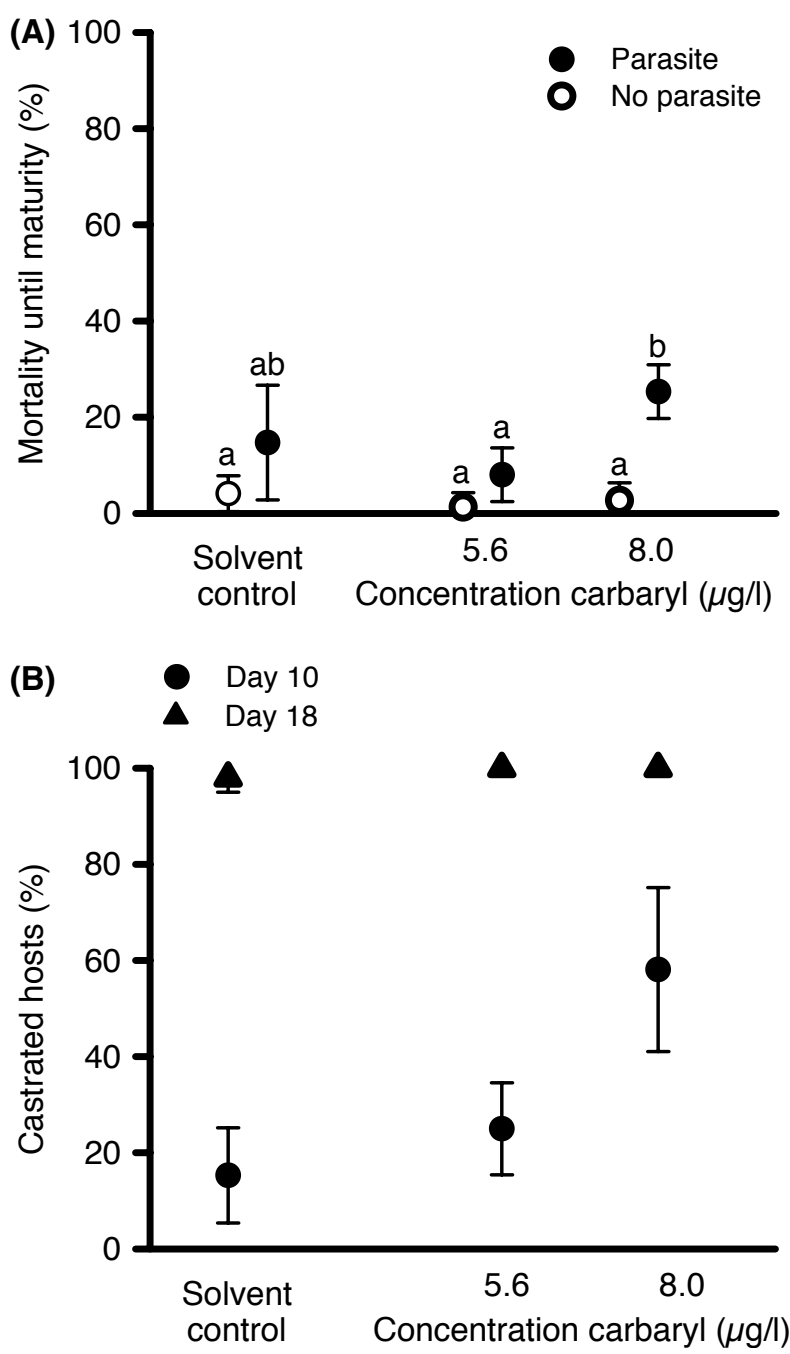

Figure 1. (A) cumulative mortality until maturity (end of juvenile phase) in the host Daphnia magna challenged by the bacterial endoparasite Pasteuria ramosa or placebo-treated and simultaneously exposed to the pesticide carbaryl or a solvent control. (B) percentage of castrated females in $P$. ramosa-challenged $D$. magna at day 10 (end of juvenile phase) and day 18 (end of experiment). Given are means and standard deviations $(n=5)$. Significantly different treatments are indicated in (A) by different letters (Bonferroni post-hoc test, $\mathrm{p}<0.01$ ).

(Table 1). Bonferroni post-hoc testing $(\mathrm{DF}=24$, mean square error $\mathrm{MSE}=0.004)$ revealed that the treatment with parasite challenge at $8.0 \mu \mathrm{g} \mathrm{l}^{-1}$ carbaryl did not significantly differ from the parasite/solvent control (Bonferroni-corrected $\mathrm{p}=0.204$ ) but did differ from all other treatments (Bonferroni-corrected $p<0.01$; Fig. 1A). Significantly different mortality was further detected between

Table 1. Results of two-way ANOVA for effects of the pesticide carbaryl and the parasite Pasteuria ramosa on mortality of the host Daphnia magna.

\begin{tabular}{lrcrc}
\hline & DF & MS & F & $p$ \\
\hline Carbaryl (C) & 2 & 0.02178 & 5.43 & 0.0113 \\
Parasite (P) & 1 & 0.13270 & 33.08 & $<0.00001$ \\
C $\times$ P & 2 & 0.01740 & 4.34 & 0.0247 \\
Residual & 24 & 0.00401 & & \\
\hline
\end{tabular}


the parasite/solvent control and the placebo/5.6 $\mu \mathrm{g} \mathrm{^{-1 }}$ carbaryl treatment (Bonferroni-corrected $\mathrm{p}=0.042$ ). All other pairwise comparisons resulted in Bonferroni-corrected p-values above 0.05 . Thus, carbaryl exposure alone had no significant effect on mortality, which showed that the experimental carbaryl concentrations were sublethal. Higher mortality in all three parasite treatments compared to their respective placebo treatments (Fig. 1A) suggested a trend to parasite-related mortality. The impact of the parasite was not significant in the absence of carbaryl, because the two solvent controls (one with and one without parasite) did not significantly differ from each other.

We observed an accelerated castration in carbarylexposed hosts (Fig. 1B). Under the given experimental conditions, all surviving daphnids either carried their first eggs at day 10 or they were already irreversibly castrated by the parasite at this time. Because almost all parasitechallenged daphnids were castrated by day 18 (Fig. 1B), castration rates at day 10 can serve as a measure for the speed with which the parasite castrated its host. Carbaryl exposure resulted in an increase of hosts already being castrated at day 10 (Fig. 1B), thus in a significant increase in the speed of castration (Table 2). Consequently, carbaryl exposure lead to a greater likelihood of zero lifetime reproduction in infected D. magna. At $8.0 \mathrm{\mu g} \mathrm{l}^{-1}$ carbaryl, the percentage of daphnids which never reproduced tripled that of the parasite/solvent control treatment.

By using offspring data from a smaller-sized preliminary test performed at a different food level in combination with the mortality and castration data of experiment 1, we calculated a rough estimate for the intrinsic rate of natural increase, $r$, according to the Euler-Lotka equation. In absence of $P$. ramosa challenge, carbaryl had no impact on $r$ (ranging from $0.311-0.312 \mathrm{day}^{-1}$ in the three treatments). The parasite reduced $r$ to $0.215 \mathrm{day}^{-1}$ in the absence of carbaryl and to 0.209 and 0.133 day $^{-1}$ at carbaryl concentrations of 5.6 and $8.0 \mathrm{\mu g} \mathrm{l}^{-1}$, respectively.

\section{Experiment 2}

As in experiment 1 , mortality occurred mainly before maturation, which started at day 6 in this experiment due to higher food levels compared to experiment 1 . Between 10 and $30 \%$ of D. magna died before reaching maturity in the low carbaryl and solvent control treatments (Fig. 2A). The mortality rate was equally low in the $8.0 \mu \mathrm{g} \mathrm{l}^{-1}$ carbaryl/parasite-free treatment but increased to $80 \%$ in the $8.0 \mu \mathrm{g} \mathrm{l}^{-1}$ carbaryl/parasite treatment (Fig. 2A). The mortality in F. magnivora-parasitized D. magna at $8.0 \mu \mathrm{g}$ $1^{-1}$ carbaryl differed significantly from the mortality of F. magnivora-free D. magna at $8.0 \mathrm{\mu g}^{-1}$ carbaryl as well as from the mortality in $F$. magnivora-parasitized $D$. magna

Table 2. Results of one-way ANOVA for effect of the pesticide carbaryl on speed of castration (\% of castrated females on day 10) in the host Daphnia magna challenged by the parasite Pasteuria ramosa.

\begin{tabular}{lrccc}
\hline & DF & MS & $F$ & $p$ \\
\hline Carbaryl & 2 & 0.25211 & 15.77 & 0.0004 \\
Residual & 12 & 0.01599 & & \\
\hline
\end{tabular}

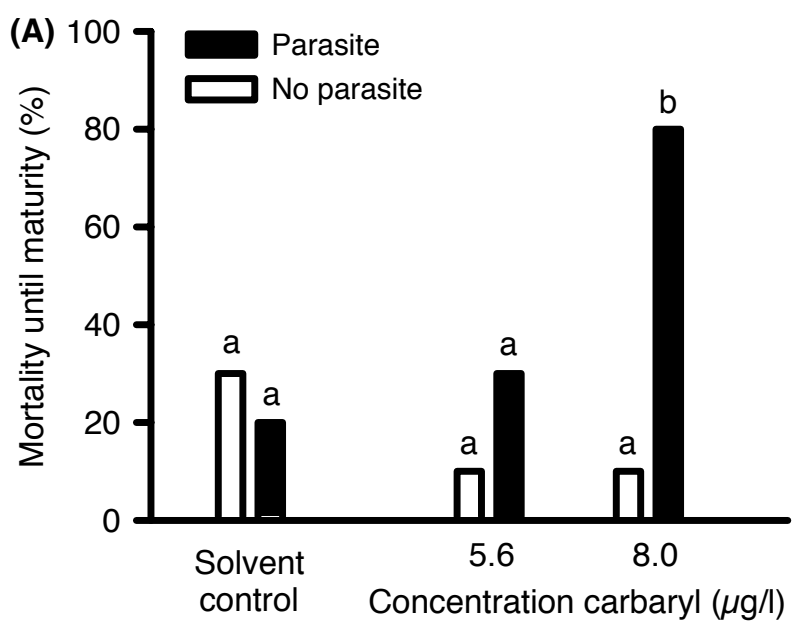

$\begin{array}{lll}\mathbf{\Delta} & \text { Parasite } \\ & \text { No parasite }\end{array}$

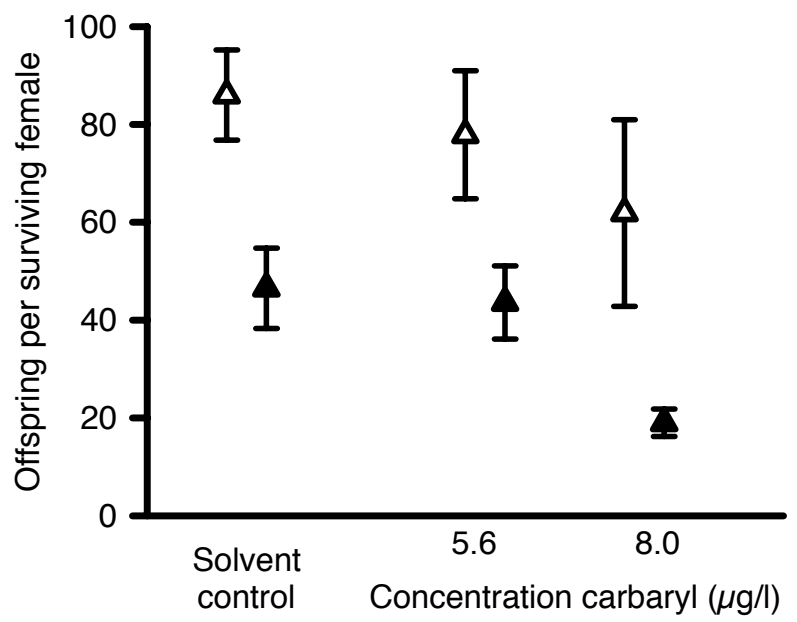

Figure 2. (A) cumulative mortality until maturity (end of juvenile phase) and (B) fecundity of surviving females until day 18 in the host Daphnia magna infected by the microsporidian endoparasite Flabelliforma magnivora or non-infected isogenic D. magna and simultaneous exposure to the pesticide carbaryl or a solvent control. Significantly different treatments are indicated in (A) by different letters (Fisher exact tests with Bonferroni correction, $\mathrm{p}<0.05)$. Given are percentages per treatment $(\mathrm{A})$ or means and standard deviations $(\mathrm{B})$.

both exposed to a lower carbaryl concentration and in the absence of carbaryl (Fisher exact tests, Bonferroni-corrected $\mathrm{p}<0.05)$. Mortality did not significantly differ among other treatments (Fig. 2A).

In contrast to $P$. ramosa, F. magnivora did not castrate its host, but reduced the number of host offspring per brood increasingly over time. F. magnivora -infected $D$. magna produced significantly fewer offspring during the experiment than the $F$. magnivora-free females and carbaryl exposure also significantly reduced the total number of living offspring (Table 3, Fig. 2B). No interaction was observed between parasite and carbaryl with regard to offspring production (Table 3).

The data on age-specific offspring production and mortality observed in experiment 2 were used to calculate the intrinsic rate of natural increase, $r$, for each treatment as 
Table 3. Results of two-way ANOVA for effects of the pesticide carbaryl and the parasite Flabelliforma magnivora on the number of offspring per surviving female in the host Daphnia magna.

\begin{tabular}{lrrrc}
\hline & DF & \multicolumn{1}{c}{ MS } & \multicolumn{1}{c}{$\mathrm{F}$} & \multicolumn{1}{c}{$\mathrm{p}$} \\
\hline Carbaryl (C) & 2 & 1540.2 & 9.83 & $<0.0001$ \\
Parasite (P) & 1 & 12022.2 & 76.72 & $<0.0001$ \\
C $\times$ P & 2 & 50.4 & 0.32 & 0.727 \\
Residual & 36 & 156.7 & & \\
\hline
\end{tabular}

an indicator for population level effects. Carbaryl slightly reduced $\mathrm{r}$ in parasite-free treatments from 0.338 day $^{-1}$ in the solvent control to $0.317 \mathrm{day}^{-1}$ at $8.0 \mathrm{\mu g} \mathrm{l}^{-1}$ carbaryl. F. magnivora reduced $\mathrm{r}$ to $0.318 \mathrm{day}^{-1}$ in the absence of carbaryl but to 0.279 and 0.126 day $^{-1}$ at carbaryl concentrations of 5.6 and $8.0 \mu \mathrm{g} \mathrm{l}^{-1}$, respectively.

\section{Discussion}

Although carbaryl is used worldwide in a great diversity of crops and in large amounts (Relyea and Mills 2001), measured environmental concentrations are usually low due to the rapid hydrolysis at $\mathrm{pH}$ values above 7 (EPA 2003). The maximum measured surface water concentration reported in the reviewed monitoring studies was $5.5 \mu \mathrm{g}$ $1^{-1}$, whereas estimated environmental peak concentrations in surface waters range from 23.3 to $152.6 \mathrm{~g} \mathrm{l}^{-1}$ for five selected crops (EPA 2003). In the present study, we used environmentally relevant carbaryl concentrations below these levels, which proved sublethal in absence of parasites. Yet, exposure to these carbaryl concentrations and simultaneous challenge by a parasite synergistically reduced survival of the host. This observation was found congruently for two parasites from two different taxa with different modes of transmission, which indicates a general pattern for interaction of carbaryl and parasitic diseases on D. magna survival. The speed of castration, and thereby host fecundity, was strongly affected by carbaryl in experiment 1 , whereas no synergistic interaction on number of offspring was observed in experiment 2. This difference may reflect the differences in physiological mechanisms by which the two parasites reduce host reproduction. In conclusion, carbaryl strongly enhanced the virulence of parasites in the host D. magna. The observed impacts on individual host fitness are expected to translate into effects on the population level, as it has been shown that the strong negative effect of parasites on individual D. magna reduces host population densities and increases the risk of extinction (Ebert et al. 2000). The much stronger reduction in the intrinsic rate of natural increase in the combined parasite/carbaryl treatment compared to the single stressor treatments in both experiments reflects the observed interactions between the two stressors and illustrates the likelihood of a synergistic impact at the population level.

Within the host model species D. magna, considerable genetic variation exists for parasite resistance (Little and Ebert 1999, Carius et al. 2001), which enables parasitemediated selection as already shown for $P$. ramosa and the fungal parasite Metschnikowia bicuspidata (Mitchell et al. 2004, Decaestecker et al. 2007, Duffy and Sivars-Becker 2007, Duncan and Little 2007). Increased parasite virulence upon carbaryl exposure suggests that the selection pressure of a parasite epidemic in a Daphnia population may be strengthened by simultaneous pollution with carbaryl, thereby leading to a change in host-parasite co-evolutionary trajectories and an intensified arms race between host and parasite.

Pasteuria ramosa is a long-lasting parasite heavily exploiting host resources, for which early castration but intermediate virulence was proposed as an optimal strategy to maximise parasite transmission success (Jensen et al. 2006). Increased host mortality, with hosts dying before even reaching adulthood, reduces production of mature $P$. ramosa transmission stages, which is positively correlated with host age (Ebert et al. 2004). In the case of $F$. magnivora, increased mortality of hosts before maturation dramatically affects vertical transmission and thereby the persistence of the parasite in the host population. Following this reasoning, increased mortality of infected hosts due to environmental pollution would not only impact the host population but also both parasites.

Assuming that the immune response of the Daphnia host is involved in determining the development of the parasitic infections, accelerated castration and increased mortality of infected hosts are symptomatic of reduced disease resistance. This suggests that the observed synergistic effect of carbaryl on disease development was due to an impairment of the host immune system, caused by an immunosuppressive activity of the pesticide. Biomarker studies using immunological parameters have already pointed to an immunomodulatory activity of carbaryl in rabbits (Street and Sharma 1975), mice (Hong et al. 2004) and earthworms (Ville et al. 1997). Yet, non-specific stress responses triggered by carbaryl exposure may also have indirectly resulted in reduced immunocompetence. Such an indirect mechanism was proposed as an explanation for the finding that mechanical stress influenced immune parameters and host-parasite interactions in molluscs (Lacoste et al. 2001, Malham et al. 2003). Possible explanations for an indirect immunosuppression by chemical stress are tradeoffs in energy allocation between immune defence responses and toxicant-induced responses (e.g. energy-consuming metabolic detoxification) or toxicant-induced reduction in food uptake resulting in impaired host condition, as it has been reported for ciliate hosts (Restif and Kaltz 2006). Whether the observed reduced disease resistance due to carbaryl exposure was indeed caused by direct interference of the pesticide with the immune system in $D$. magna requires confirmation by measuring functional immune responses. Yet, at present it is not known which specific mechanisms mediate resistance of D. magna towards P. ramosa (Mucklow et al. 2004) and identifying them was beyond the scope of the present study.

Increased impact of infectious diseases in natural populations caused by environmental pollution implies that pollutants can suppress immunocompetence at environmentally relevant concentrations and that this immunosuppression leads, directly or indirectly, to enhanced susceptibility to diseases. Field and laboratory studies based on selected immune defence functions such as phagocytosis, lysozyme levels, and release of reactive oxygen metabolites have reported impaired immunocompetence in invertebrates due to pollutants from a variety of classes such as 
heavy metals, polychlorinated biphenyls, polyaromatic hydrocarbons, and pesticides (Galloway and Depledge 2001). However, direct causal links between suppression of single immune response parameters and enhanced infectious disease impacts are mostly lacking for invertebrates, obscuring conclusions of cause-effect relationships between environmental pollution and increased impact of infectious diseases (Galloway and Depledge 2001). Whereas many studies investigated pollutant-mediated impacts on host resistance using pre-infected hosts sampled in the field, so far in only one study an invertebrate host has been experimentally infected during toxicant exposure (Chu and Hale 1994). Only such an experimental set-up includes the infection phase as well as early stages of the disease development in the assessment and excludes uncontrolled confounding factors such as field conditions during the actual infection phase. Chu and Hale (1994) reported enhanced disease progression in the eastern oyster Crassostrea virginica exposed to water extracts of contaminated sediments, but the causative agent could not be identified because the tested sediments were contaminated with a variety of pollutants.

The present study provides evidence for suppressed disease resistance in an invertebrate host model caused by a specified pollutant and is the first to demonstrate in vivo effects on parasite virulence by environmentally relevant concentrations of a pesticide. Thus, synergistic interactions between pesticides and in situ expression of infectious diseases can occur at pesticide concentrations likely to be encountered in the aquatic environment. Together with the previously reported synergistic interactions between carbaryl and predator presence (Relyea and Mills 2001), our results imply that fundamentally different biotic antagonists such as predators and parasites may boost effects of pollutants and, vice versa, that the impact of natural antagonists can be boosted by pollutant exposure. Given the ubiquity of pesticide usage and the commonness of parasite presence with the potential of rapidly developing epidemics, synergistic effects such as reported here are expected to facilitate strong impacts of pollutants in natural populations. This further substantiates the previously expressed need for considering biotic stressors such as the presence of predators and parasites when aiming at an ecologically more realistic risk assessment of anthropogenic substances (Heinonen et al. 1999, Relyea and Mills 2001). Currently, the ecological risk assessment of anthropogenic substances relies at least partly on extrapolating results from standard laboratory tests, which assess effects on organisms generally in the absence of their antagonists. Uncertainty factors are applied on the results of these standard tests to account for extrapolation across species and from individuals to populations in order to predict no-effect levels for field populations (Stark et al. 2004). Yet, it is questionable whether the presently used uncertainty factors are large enough to additionally cover synergistic interaction effects between pollutants and various natural antagonists.

The issue of interactions between environmental pollutants and parasitic infection is not restricted to the specific model used here but will most likely be relevant to other host-parasite systems. However, the host model Daphnia magna is shown here to be a promising tool for investigating potential impacts of pollutants and other stressors on host resistance in invertebrates, which might help in progressing towards a more comprehensive assessment of effects of anthropogenic substances on natural populations.

Acknowledgements - We thank Joost Vanoverbeke for practical help. This research was supported by a postdoctoral fellowship grant from the K. U. Leuven to AC, by a postdoctoral research grant from the F.W.O.-Vlaanderen to $\mathrm{ED}$, by a grant of the Institute for the Promotion of Innovation by Science and Technology in Flanders (IWT) to MJ, and by project OT/04/23 and GOA/08/06 of the K.U.Leuven Research Fund.

\section{References}

Anderson, R. M. and May, R. M. 1979. Population biology of infectious diseases. Part I. - Nature 280: 361-367.

Bull, J. J. 1994. Virulence. - Evolution 48: 1423-1437.

Carius, H. J. et al. 2001. Genetic variation in a host-parasite association: potential for coevolution and frequency-dependent selection. - Evolution 55: 1136-1145.

Chu, F.-L. E. and Hale, R. C. 1994. Relationship between pollution and susceptibility to infectious disease in the eastern Oyster, Crassostrea virginica. - Mar. Environ. Res. 38: 243256.

Decaestecker, E. et al. 2004. Haunted by the past: evidence for dormant stage banks of microparasites and epibionts of Daphnia. - Limnol. Oceanogr. 49: 1355-1364.

Decaestecker, E. et al. 2007. Host-parasite Red Queen dynamics archived in pond sediment. - Nature 450: 870-874.

Descotes, J. 2006. Methods of evaluating immunotoxicity. - Expert. Opin. Drug Metabol. Toxicol. 2: 249-259.

Duffy, M. A. and Sivars-Becker, L. 2007. Rapid evolution and ecological host-parasite dynamics. - Ecol. Lett. 10: 44-53.

Duncan, A. B. and Little, T. J. 2007. Parasite-driven genetic change in a natural population of Daphnia. - Evolution 61: 796-803.

Ebert, D. 1994. Virulence and local adaptation of a horizontally transmitted parasite. - Science 265: 1084-1086.

Ebert, D. 2005. Ecology, epidemiology, and evolution of parasitism in Daphnia, - Natl Library of Medicine (US), Natl Center for Biotechnology Information, Bethesda, MD. Available from: <http://www.ncbi.nlm.nih.gov/entrez/query.fcgi? $\mathrm{db}=$ Books $>$.

Ebert, D. et al. 2000. The effect of parasites on host population density and extinction: experimental epidemiology with Daphnia and six microparasites. - Am. Nat. 156: 459-477.

Ebert, D. et al. 2001. Temporal and spatial dynamics of parasite richness in a Daphnia metapopulation. - Ecology 82: 34173434.

Ebert, D. et al. 2004. The evolution of virulence when parasites cause host castration and gigantism. - Am. Nat. 164: S19S32.

EPA 2003. Environmental fate and ecological risk assessment for the re-registration of carbaryl. - US Environ. Protection Agency, Washington, DC.

Galloway, T. S. and Depledge, M. H. 2001. Immunotoxicity in invertebrates: measurement and ecotoxicological relevance. - Ecotoxicology 10: 5-23.

Green, J. 1974. Parasites and epibionts of Cladocera. - Trans. Zool. Soc. Lond. 32: 417-515.

Heinonen, J. et al. 1999. The effects of parasites and temperature on the accumulation of xenobiotics in a freshwater clam. - Ecol. Appl. 9: 475-481.

Hong, C.-C. H. et al. 2004. Effect of endocrine disrupting chemicals on lipopolysaccharide-induced tumor necrosis 
factor-alpha and nitric oxide production by mouse macrophages. - Biol. Pharm. Bull. 27: 1136-1139.

Jensen, K. H. et al. 2006. - Empirical support for optimal virulence in a castrating parasite. PLoS Biol. 4: 1265-1269.

Jokela, J. et al. 2005. Virulence of parasites in hosts under environmental stress: experiments with anoxia and starvation. - Oikos 108: 156-164.

Klüttgen, B. et al. 1994. ADaM, an artificial freshwater for the culture of zooplankton. - Water Res. 28: 743-746.

Koller, L. D. 2001. A perspective on the progression of immunotoxicology. - Toxicology 160: 105-110.

Lacoste, A. et al. 2001. Stress and stress-induced neuroendocrine changes increase the susceptibility of juvenile oysters (Crassostrea gigas) to Vibrio splendidus. - Appl. Environ. Microb. 67: 2304-2309.

Little, T. J. and Ebert, D. 1999. Associations between parasitism and host genotype in natural populations of Daphnia (Crustaceae: Cladocera). - J. Anim. Ecol. 68: 134-149.

Malham, S. K. et al. 2003. Evidence for a direct link between stress and immunity in the mollusc Haliotis tuberculata. - J. Exp. Zool. 295A: 136-144.

Mangin, K. L. et al. 1995. Virulence and transmission modes of two microsporidia in Daphnia magna. - Parasitology 111: 133-142.

Minchella, D. J. and Scott, M. E. 1991. Parasitism: a cryptic determinant of animal community structure. - Trends Ecol. Evol. 6: 250-254.

Mitchell, S. E. et al. 2004. The effect of a pathogen epidemic on the genetic structure and reproductive strategy of the crustacean Daphnia magna. - Ecol. Lett. 7: 848-858.
Mucklow, P. T. et al. 2004. Variation in phenoloxidase activity and its relation to parasite resistance within and between populations of Daphnia magna. - Proc. R. Soc. Lond. B 271: $1175-1183$

Relyea, R. A. and Mills, N. 2001. Predator-induced stress makes the pesticide carbaryl more deadly to gray treefrog tadpoles (Hyla versicolor). - Proc. Natl Acad. Sci. USA 98: 2491-2496.

Restif, O. and Kaltz, O. 2006. Condition-dependent virulence in a horizontally and vertically transmitted bacterial parasite. - Oikos 114: 148-158.

Rohr, J. R. et al. 2006. Community ecology as a framework for predicting contaminant effects. - Trends Ecol. Evol. 21: 606613.

Stark, J. D. et al. 2004. How risky is risk assessment: the role that life history strategies play in susceptibility of species to stress. - Proc. Natl Acad. Sci. USA 101: 732-736.

Stirnadel, H. A. and Ebert, D. 1997. Prevalence, host specificity and impact on host fecundity of microparasites and epibionts in three sympatric Daphnia species. - J. Anim. Ecol. 66: 212222.

Street, J. C. and Sharma, R. P. 1975. Alteration of induced cellular and humoral immune responses by pesticides and chemicals of environmental concern: quantitative studies of immunosuppression by DDT, aroclor 1254, carbaryl, carbofuran, and methylparathion. - Toxicol. Appl. Pharm. 32: 587-602.

Thompson, J. N. 2005. The geographic mosaic of coevolution. - Univ. of Chicago Press.

Ville, P. et al. 1997. Immuno-modulator effects of carbaryl and 2,4 D in the earthworm Eisenia foetida andrei. - Arch. Environ. Contam. Toxicol. 32: 291-297. 